\title{
Virtual and Augmented Reality, from Design to Evaluation
}

\author{
Rachid Gherbi ${ }^{(凶)}$ \\ Université de Paris-Sud XI, Orsay, France \\ rachid.gherbi@u-psud.fr
}

\begin{abstract}
The talk will highlight the general idea of Virtual and Augmented Reality (V\&AR), from design specifications to evaluation methods. After a short introduction into the history of $\mathrm{V} \& \mathrm{AR}$, we will have a look at various types and concepts of V\&AR known today. We then discuss some experimental cases to highlight the need of specification of all V\&AR parts, such as technical and hardware constraints but also those concerning tasks modeling and users needs. We will present some criteria we consider when evaluating a V\&AR experiences success. Finally, some Algerian V\&AR applications and projects will also be presented in order to show national developments in this area.
\end{abstract}

